\title{
A First Look at Picking Dual Variables for Maximizing Reduced Cost Fixing
}

\author{
Omid Sanei Bajgiran, Andre A. Cire, and Louis-Martin Rousseau
}

Version Post-print/accepted manuscript

Citation Bajgiran O.S., Cire A.A., Rousseau LM. (2017) A First Look at Picking (published version) Dual Variables for Maximizing Reduced Cost Fixing. In: Salvagnin D., Lombardi M. (eds) Integration of AI and OR Techniques in Constraint Programming. CPAIOR 2017. Lecture Notes in Computer Science, vol 10335. Springer, Cham.

Publisher's Statement The final publication is available at link.springer.com via http://dx.doi.org/10.1007/978-3-319-59776-8_18.

\section{How to cite TSpace items}

Always cite the published version, so the author(s) will receive recognition through services that track citation counts, e.g. Scopus. If you need to cite the page number of the author manuscript from TSpace because you cannot access the published version, then cite the TSpace version in addition to the published version using the permanent URI (handle) found on the record page.

This article was made openly accessible by $U$ of $T$ Faculty. Please tell us how this access benefits you. Your story matters. 


\title{
A First Look at Picking Dual Variables for Maximizing Reduced Cost Fixing
}

\author{
Omid Sanei Bajgiran ${ }^{1,3}$, Andre A. Cire ${ }^{1}$, and Louis-Martin Rousseau ${ }^{2,3}$ \\ ${ }^{1}$ Department of Management, University of Toronto Scarborough \\ ${ }^{2}$ Department of Mathematics and Industrial Engineering, Polytechnique Montréal \\ ${ }^{3}$ Interuniversity Research Center on Enterprise Networks, Logistics and \\ Transportation (CIRRELT)
}

\begin{abstract}
Reduced-cost-based filtering in constraint programming and variable fixing in integer programming are techniques which allow to cut out part of the solution space which cannot lead to an optimal solution. These techniques are, however, dependent on the dual values available at the moment of pruning. In this paper, we investigate the value of picking a set of dual values which maximizes the amount of filtering (or fixing) that is possible. We test this new variable-fixing methodology for arbitrary mixed-integer linear programming models. The resulting method can be naturally incorporated into existing solvers. Preliminary results on a large set of benchmark instances suggest that the method can effectively reduce solution times on hard instances with respect to a state-of-the-art commercial solver.
\end{abstract}

Keywords: mixed-integer programming; Variable Fixing Methodology; Reduced-cost based filtering

Pre-print - CPAIOR'17.

DOI: https://doi.org/10.1007/978-3-319-59776-8_18

\section{Introduction}

A key feature of modern mathematical programming solvers refers to the wide range of techniques that are applied to simplify an instance. Typically considered during a preprocessing stage, these techniques aim at fixing variables, eliminating redundant constraints, and identifying structure that can either lead to speedups in solution times or provide useful information about the model at hand. Examples of valuable information include, e.g., potential numerical issues or which subset of inequalities and variables may be responsible for the infeasibility [12], if that is the case. These simplification methods alone reduce solution times by half in state-of-the-art solvers such as CPLEX, SCIP, or Gurobi [4], thereby constituting an important tool in the use of mixed-integer linear programming (MILP) in practical real-world problems [11].

In this paper we investigate a new simplification technique that expands upon the well-known reduced cost fixing method, first mentioned by Balas and Martin [2] and largely used both in the mathematical programming and the constraint programming (CP) communities. The underlying idea of the method is 
straightforward: Given a linear programming (LP) model and any optimal solution to such a model, the reduced cost of a variable indicates the marginal linear change in the objective function when the value of the variable in that solution is increased [5]. In cases where the LP encodes a relaxation of an arbitrary optimization problem, we can therefore filter all values from a variable domain that, based on the reduced cost, incur a new objective function value that is worse than a known solution to the original problem. The result is a tighter variable bound which can then trigger further variable fixing and other simplifications.

This simple but effective technique is widely applied in MILP presolving $[4,11,12]$ and plays a key role in a variety of propagation methods for global constraints in CP $[7,9,8]$. It can be easily incorporated into solvers since the reduced costs are directly derived from any optimal set of duals, which in turn can be efficiently obtained by solving an LP once. The technique is also a natural way of exploiting the strengths of MILP within a CP framework, since the dual values incorporate a global bound information that is potentially lost when processing constraints one at a time (a concept that is explored, e.g., in $[16,18,3]$ ).

However, in all cases typically only one reduced cost per variable is considered, that is, the one obtained after solving the LP relaxation of a MILP. In theory, any set of feasible duals provides valid reduced costs that may lead, in turn, to quite different variable bound tightenings. This question was originally raised by Sellmann [15], who demonstrated that not only distinct dual vectors would result in significantly different filtering behaviors, but that potentially sub-optimal dual vectors could yield much more pruning than the optimal ones.

Our goal in this work is to investigate the potential effect of picking the dual vector that maximizes reduced-cost-based filtering. By doing so, we revisit the notion of relaxed consistency for reduced costs fixing; that is, we wish to influence the choice of the dual values given by a relaxation so as to maximize the amount of pruning that can be performed. We view the proposed techniques as a first direction towards answering some of the interesting questions raised in the field of CP-based Lagrangian relaxation [15,3], in particular related to how to select the dual variables (or, equivalently, the Lagrangian multipliers) to maximize propagation.

The contribution of this paper is to formulate the problem of finding the dual vectors that maximize the number of reductions as an optimization problem defined over the space of optimal (or just feasible) dual values. We compare this approach to achieving full relaxed consistency, which can be obtained by solving a large (but polynomial) number of LP problems. The resulting technique can be seamlessly incorporated into existing solvers, and preliminary results over the MIPLIB indicate that it can significantly reduce solution time as well as the size of the branching tree when proving the optimality of a primal bound. We hope to motivate further research on the quality of the duals used within both ILP and $\mathrm{CP}$ technology.

For the sake of clarity and without loss of generality, the proposed approaches will be detailed in the context of integer linear programs (ILPs), i.e., where all variables are integers, as opposed to mixed-integer linear programming models. 
This technique is also applicable in the context of $\mathrm{CP}$, if one can derive a (partial) linear relaxation of the model [14].

The paper is organized as follows. Section 2 introduces the necessary notation and the basic concepts of reduced cost fixing and the related consistency notions. Next, we discuss one alternative to obtain an approximate consistency in Section 3. Finally, we present a preliminary numerical study in Section 4 and conclude in Section 5.

\section{Reduced Cost Fixing \& Relaxed-Consistency}

For the purposes of this paper, consider the problem

$$
z_{P}:=\min \left\{c^{T} x: A x \geq b, x \geq 0\right\}
$$

with $A \in \mathbb{R}^{n \times m}$ and $b, c \in \mathbb{R}^{n}$ for some $n, m \geq 1$. We assume that (P) represents the LP relaxation of an ILP problem $\mathrm{P}_{S}$ with an optimal solution value of $z^{*} \geq z_{P}$ and where variables $\left\{x_{i}: i \in S\right\}$ are subject to integrality constraints. The dual of the problem $(\mathrm{P})$ can be written as

$$
z_{D}:=\max \left\{u^{T} b: u^{T} A \leq c^{T}, u \geq 0\right\}
$$

where $u \in \mathbb{R}^{m}$ is the vector of dual variables. We assume for exposition that $\mathrm{P}_{S}$, $(\mathrm{P})$, and (D) are bounded (the results presented here can be easily generalized when that is not the case).

We have $z_{P}=z_{D}$ (strong duality) and for every optimal solution $x^{*}$ of (P), there exists an optimal solution $u^{*}$ to (D) such that $u^{* T}\left(b-A x^{*}\right)=0$ (complementary slackness). Moreover, for some $j$ such that $x_{j}^{*}=0$, the quantity

$$
\bar{c}_{j}=c_{j}-u^{* T} A_{j}
$$

is the reduced cost of variable $x_{j}$ and yields the marginal increase in the objective function if $x_{j}^{*}$ moves away from its lower bound. Thus, if a given known feasible solution with value $z^{U B} \geq z^{*}$ is available to the original ILP, the reduced cost fixing technique consists of fixing $x_{j}^{*}=0$ if

$$
z_{P}+\bar{c}_{j} \geq z^{U B},
$$

since any solution with $x_{j}^{*}>0$ can never improve upon the existing upper bound $z^{U B}$. We remark in passing that the condition (RCF) can be generalized to establish more general bounds on a variable. That is, we can use the reduced cost $\bar{c}_{j}$ to deduce values $l_{j}$ and $u_{j}$ such that either $x_{j}^{*} \geq l_{j}$ or $x_{j}^{*} \leq u_{j}$ in any optimal solution (see, e.g., [10]). In this paper we restrict our attention to the classical case described above. We refer to Wolsey [17] and Nemhauser \& Wolsey [13] for the formal proofs of correctness. 
The dual variables $u^{*}$ for the computation of $(\mathrm{RC})$ can be obtained with very little computational effort after finding an optimal solution $x^{*}$ to $(\mathrm{P})$ (e.g., they are computed simultaneously to $x^{*}$ when using the Simplex method). In the most of practical known implementations concerning ILP presolving and CP propagation methods, the reduced cost fixing is typically carried out using the single $u^{*}$ computed after solving every LP relaxation $[7,12]$. Note, however, that (D) may contain multiple optimal solutions, each potentially yielding a different reduced cost $\bar{c}_{j}$ that may or may not satisfy condition (RCF).

One therefore does not need to restrict its attention to a unique $u^{*}$, and can potentially improve the number of variables that are fixed if the whole dual space is considered instead. This would mean that if there exists a reduced cost $\bar{c}_{j}$ such that variable $x_{j}$ can be fixed to 0 with respect to $z^{U B}$, then there exist a dual vector $u$ such that $u^{T} b+\left(c_{j}-u^{T} A_{j}\right) \geq z^{U B}$. This was first demonstrated by [15] in the context of CP-based Lagrangian Relaxation, which also pointed out that often more filtering occurs when $u$ is not an optimal dual vector and therefore we might have that $u^{T} b<z_{P}$.

Achieving the notion of Relaxed Consistency, as defined in [6], is thus quite consuming in such a condition. This form of consistency can be casted, in the context of ILP, as follows.

Definition 1. Let $P_{S}$ be an ILP model with linear programming relaxation $(P)$ and its corresponding dual $(D)$. The model $P_{S}$ is relaxed consistent (or relaxed$P$-consistent) with respect to an upper bound $z^{U B}$ to $P_{S}$ if for any dual feasible $u^{*}$ to $(D)$ and its associated reduced cost $\bar{c}$ vector, condition $(R C F)$ is never satisfied, i.e., $z_{P}+\bar{c}_{j}<z^{U B}$ for all $j=1, \ldots, n$.

If a model is relaxed consistent according to Definition 1, then it is not possible to fix any variable $x_{j}$ via reduced costs.

Consistent with the theory presented in $[6,15]$, any ILP formulation can be efficiently converted into a relaxed consistent formulation in polynomial time. Given an ILP model $\mathrm{P}_{S}$ and the primal (P) and dual (D) of its associated linear programming relaxation, the set of optimal dual solution coincides with the polyhedral set $\mathcal{D}=\left\{u \in \mathbb{R}^{m}: u^{T} A \geq c, u \geq 0\right\}$. Thus, a variable $x_{j}$ can be fixed to zero if the optimal solution $\bar{c}_{j}^{*}$ of the problem $\bar{c}_{j}^{*}=\max \left\{c_{j}-u^{T} A_{j}: u \in \mathcal{D}\right\}$ is such that $z_{P}+\bar{c}_{j}^{*} \geq z^{U B}$. This means that the complexity of the procedure is dominated by the cost of solving $O(n)$ LP models, each of which can be done in weakly polynomial time [17].

\section{Dual Picking for Maximum Reduced Cost Fixing}

Establishing relaxed consistency by solving $O(n)$ LP models is impractical when a model has any reasonably large number of variables. We thus propose a simple alternative model that exploits the underlying concept of searching in the dual space for maximizing filtering. Namely, as opposed to solving an LP for each variable, we will search for the dual variables that together maximize the number of variables that can be fixed. This can be written as the following MILP model: 


$$
\begin{array}{ll}
\max & \sum_{i=1}^{n} y_{i} \\
\text { s.t. } & u^{T} A \leq c \\
& u^{T} b=z_{P} \\
& u^{T} b+\left(c_{j}-u^{T} A_{j}\right) \geq z^{U B}-\left(\mathbf{1}-y_{j}\right) M \quad \forall j \\
& u \geq 0 \\
& y \in\{0,1\}^{n}
\end{array}
$$

In the model (DP-RCF) above, we are searching for the dual variables $u$, on the optimal dual face, that maximize the number of variables fixed. Specifically, we will define a binary variable $y_{i}$ in such a way that $y_{i}=1$ if and only if we fix it allows to deduce that $x_{j}$ can be fixed to 0 .

To enforce this, let $M$ be a sufficiently large number, and $\mathbf{1}$ an n-dimensional vector containing all ones. Constraints (1), and (4) ensure that $u^{*}$ is dual feasible. If $y_{i}=1$, then inequality (3) reduces to condition (RCF) and the associated $x_{i}$ should be fixed to 0. Otherwise, the right-hand side of (3) is arbitrary small (in particular to account for arbitrarily small negative reduced costs). Constraint (2) enforces strong duality and the investigation of optimal dual vectors only, it can be omitted in order to explore the whole dual feasible space (as sub-optimal dual vectors can perhaps filter more [15]). Finally, constraint (5) defines the domain of the $y$ variable and the objective maximizes the number of variables fixed.

The model (DP-RCF) does not necessarily achieve relaxed consistency as it yields a single reduced cost vector and it is restricted to the optimal dual face. However, our experimental results indicate that the model can be solved quite efficiently and yields interesting bounds. Notice also that any feasible solution to (DP-RCF) corresponds to a valid set of variables to fix, and hence any solutions found during search can be used to our purposes as well.

\section{Preliminary Numerical Study}

We present a preliminary numerical study of our technique on a subset of the MIPLIB 2010 benchmark [1]. All experiments were performed using IBM ILOG CPLEX 12.6.3 on a single thread of an Intel Core i7 CPU 3.40GHz with 8.00 GB RAM.

Our goal for these experiments is twofold: We wish first to evaluate the filtering achieved by the dual picking models (DP-RCF) in comparison to the full relaxed-consistent model, and next verify what is the impact of fixing these variables in CPLEX when proving optimality. For the first criteria, we considered the number of fixed variables according to three different approaches:

1. The model (DP-RCF) with a time limit of 10 minutes, denoted by DP.

2. A modified version of the model (DP-RCF) without constraint (2), i.e., we increased our search space by considering any feasible dual solution, also fixing a time limit of 10 minutes. We denote this approach by DP-M. 
3. Solving the $O(n)$ LP models to achieve relaxed consistency in view of Definition 1, with no time limit. This method provides the maximum number of variables that can be fixed thought RCF and thus an upperbound for both DP and DP-M, it is denoted by RCC (relaxed reduced-cost consistency).

In all the cases above, we considered the optimal solution value of the instance as the upper bound $z^{U B}$ for the (RCF) condition, which results in the strongest possible filtering for a fixed set of duals.

Next, to assess the impact of fixing variables in the ILP solution process, we ran the default CPLEX for each approach above, specifically setting the fixed variables to zero and providing the optimal solution value of each instance to the solver. We have also ran default CPLEX without any variable fixing and with the optimal value as an input, in order to evaluate the impact of variable fixing in proving the optimality of a particular bound.

As a benchmark we considered all "easy" instances of the MIPLIB that could be solved within our memory or limit of $8 \mathrm{~GB}$ and a time limit of $60,000 \mathrm{~s}$. We have also eliminated all instances where relaxed consistency could not fix any variable. This resulted in 36 instances.

The results are depicted in Table 1, where Aux stands for the CPU time required to solve the auxiliary model (DP-RCF) and Vars is the number of variables which could be fixed. Moreover, Cons and Vars in the Dimension category indicate the number of constraints and decision variables of each instance, respectively, and DC shows the number of variables that default CPLEX can fix as a result of the final dual solution calculated by ourselves. Omitted rows indicates the auxiliary problem reached its time limit. Due to space restrictions, instances neos-16..., neos-47.., neos-93.., neos-13..., neos-84..., rmatr-p5, rmatr-p10, core253..., neos-93..., and sat... represent instances neos-1601936, neos-476283, neos-934278, neos-1337307, neos-849702, rmatr100-p5, rmatr100p10, core2536-691, neos-934278, and satellites1-25, respectively.

We first notice that achieving full relaxed consistency is quite time consuming and not practical with respect to the default solution time of CPLEX. When looking at both DP models, it is obvious that restricting the search to the optimal dual face, rather than the whole dual feasible space, yields practically the same amount of filtering while being orders of magnitude faster. In fact, in many cases the (DP-RCF) model can be solved in less than a second.

To determine whether such filtering is worth the extra effort, we compare the solution time of DP against the default solution time of CPLEX. For each instance we compute the speedup as the solution time of CPLEX divided by the sum of both solution and dual picking (i.e., solving (DP-RCF)) time of DP. We then compute the geometric mean of all speedups, which yields an average speed up of $20 \%$ when using our dual picking methodology over the default CPLEX.

\section{Conclusion}

In this paper, we revisited the notion of reduced-cost based filtering and variable fixing, which are known to be dependent on the available dual information. 
We defined the problem of identifying the set of dual values that maximize the number of variables which can be fixed as an optimization problem. We demonstrated that looking for a good set of such dual on the optimal dual face is considerably faster and filter almost as many variables as when considering the full feasible dual space. In many cases fixing more variable lead to a reduced search tree that can be explored faster. However, in a good number of cases, solution time increases when more variables are fixed, which is probably due to the fact that early in the tree the search takes a different path.

Future research will consider dual picking during search, so as to try to fix variables when the relative gap becomes small enough in a subtree, as well as applying the techniques in the context of constraint programming.

Table 1: General Results

\begin{tabular}{|c|c|c|c|c|c|c|c|c|c|c|c|c|c|c|c|c|c|}
\hline \multirow[b]{2}{*}{ Instance } & \multicolumn{2}{|c|}{\begin{tabular}{|l|l} 
Dimension \\
\end{tabular}} & \multirow{2}{*}{$\begin{array}{l}\text { DC } \\
\text { Vars } \\
\end{array}$} & \multirow{2}{*}{\multicolumn{2}{|c|}{\begin{tabular}{|l|} 
CPLEX Default \\
Time Nodes
\end{tabular}}} & \multicolumn{4}{|c|}{\begin{tabular}{|c|} 
DP \\
\end{tabular}} & \multicolumn{4}{|c|}{ 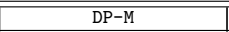 } & \multicolumn{4}{|c|}{ RCC } \\
\hline & Cons & Vars & & & & Time & Nodes & Aux & Vars & Time & Nodes & Aux & Vars & Time & Nodes & s Aux & Vars \\
\hline \multicolumn{18}{|c|}{ MILP instances } \\
\hline $30 \mathrm{n} 20 \mathrm{~b} 8$ & 578 & 18380 & 0 & 3 & 260 & 3 & 260 & 0.5 & 7282 & & & & & 3 & 90 & 494 & 13603 \\
\hline & 142 & 2728 & 33 & 187 & 17461 & 1961 & 200477 & 0.03 & 499 & & & & & 281 & 21635 & 42 & 532 \\
\hline binkar & 026 & 2298 & 165 & 7 & 1567 & 8 & 2135 & 0.1 & 200 & & & & & 5 & 902 & 51 & 281 \\
\hline co & 2539 & 15293 & 2494 & 38304 & & 2414 & 8816 & 600 & 3046 & & & & & 4464 & 72010 & 79173 & 4818 \\
\hline & 1203 & 7328 & 219 & 942 & & 257 & 1107 & 52 & 429 & & & & & 204 & 1089 & 18220 & 1540 \\
\hline & & 120 & & 68 & & 68 & 351765 & 0.02 & 0 & 124 & $\approx 6 \mathrm{e} 5$ & 9 & 485 & 26 & 94209 & 19 & 493 \\
\hline & & 25 & 50 & 1 & & 0.7 & 1115 & 0.01 & 50 & 0.7 & 1115 & 0.5 & 50 & 0.7 & 1115 & 0.8 & 50 \\
\hline & & 102 & 29 & 19 & & 17 & 46 & 26 & 213 & & & & & 26 & 329 & 18679 & 678 \\
\hline & 20 & & 8 & & & 5 & & 1 & 8 & 5 & 399 & 68 & 8 & 6 & 7 & 588 & 179 \\
\hline nec & & 44 & 867 & 327 & & 137 & 2259 & 2 & 1766 & & & & & 377 & 7549 & 7747 & 1782 \\
\hline & & 119 & & & & 195 & 1129 & 2 & 69 & & & & & 195 & 1129 & 38293 & 69 \\
\hline & & 23123 & 15843 & & & 74 & 12 & 3 & 15981 & & & & & 132 & 39 & 86254 & 16088 \\
\hline & & 141 & 0 & & & 16 & 50 & 1 & 32 & & & & & 150 & 880 & 3057 & 334 \\
\hline & & & 2 & & & 21 & & 0.08 & 286 & 21 & 860 & 107 & 286 & 32 & 1465 & 1286 & 592 \\
\hline & & & 0 & & & 74 & & 0.2 & 850 & 217 & 31211 & 952 & 853 & 110 & 5203 & 65 & 915 \\
\hline & & & 0 & & & 0.4 & & 0.05 & 0 & 0.4 & 117 & 6 & 0 & 1 & 580 & & 160 \\
\hline & & & & 2 & & 27 & 345 & 0.2 & 100 & & & & & 27 & 345 & 3260 & 100 \\
\hline & & & & & & 5 & 6 & 2 & 10 & & & & & 5 & 6 & 9601 & 100 \\
\hline & & & 0 & & & 4475 & 54077 & 0.3 & & & & & & 531 & & 35 & 553 \\
\hline & & & 0 & & & 2645 & 1770 & 0.08 & 120 & & & & & 1015 & 61271 & 50 & 149 \\
\hline & & 9 & 99 & & & 25 & & 2 & $24 \mathrm{~S}$ & & & & & 11 & 758 & 13845 & 4989 \\
\hline & & 168 & & & & & & 1.5 & 285 & & & & & 204 & 18659 & $\begin{array}{l}9 \\
96\end{array}$ & 524 \\
\hline UIMLADS 111 & & & & & 2393 & $\approx 1 \mathrm{e} 6$ & 0.02 & 13 & 2393 & $\approx 1 \mathrm{e} 6$ & & 13 & 2393 & $\approx 1 \mathrm{e} 6$ & 1 & 13 & \\
\hline \multicolumn{18}{|c|}{ Binary-Only Problems } \\
\hline acc-t & & & 1339 & 97 & & 97 & 2262 & 0 & 1339 & 97 & 2262 & 0 & 1339 & 97 & 2262 & & 1339 \\
\hline & & & 0 & 7 & & 7 & & 6 & 64 & & & & & 5 & 54 & & 126 \\
\hline & 4964 & 216 & 0 & 8313 & & 2903 & & 369 & & & & & & 883 & 7904 & 18563 & 2309 \\
\hline & & & 100 & & & & & 6 & & & & & & 0.3 & 305 & & 4013 \\
\hline & & & & & & & 216 & 1 & & & & & & 84 & 10309 & 11 & 588 \\
\hline & & 221 & 4755 & 57917 & & 57017 & $\approx 2$. & 1 & 4755 & & & & & 3518 & 83900 & 38 & 6372 \\
\hline & & & & 9 & & 6 & 110 & 5 & 77 & & & & & 5 & 30 & 10 & 150 \\
\hline & & & 0 & 0 . & 1 & 0.4 & 1 & 0.08 & & & & & & 0.4 & 1 & 142 & 84 \\
\hline & & 17 & 1737 & 10 & & 105 & & 0 & 1737 & 105 & 8223 & 0 & 1737 & 105 & 8223 & 0 & 1737 \\
\hline & & & 0 & 8 & & 5 & & 0.25 & 267 & & & & & 1 & 1 & 56 & 716 \\
\hline & & & 0 & 230 & & 230 & & 1 & 0 & 230 & 100 & 35 & ( & 25 & 260 & 2484 & 459 \\
\hline & 70 & $10 s$ & 0 & 69 & & 31 & 5920 & 0.25 & 1 & 31 & 5920 & 10 & - & 52 & 10497 & 116 & 66 \\
\hline sp98ic & 825 & 10894 & 6902 & 29041 & 149629 & 40306 & 466555 & 62 & 7098 & & & & & 345 & 35647 & $7 \quad 4724$ & 8488 \\
\hline
\end{tabular}

\section{References}

1. MIPLIB2010, http://miplib.zib.de/miplib2010-benchmark.php.

2. Egon Balas and Clarence H. Martin. Pivot and complement-a heuristic for 0-1 programming. Management Science, 26(1):86-96, 1980.

3. David Bergman, Andre A. Cire, and Willem-Jan van Hoeve. Improved Constraint Propagation via Lagrangian Decomposition, pages 30-38. Springer International Publishing, Cham, 2015. 
4. E Robert Bixby, Mary Fenelon, Zonghao Gu, Ed Rothberg, and Roland Wunderling. MIP: Theory and practice - closing the gap. In System modelling and optimization, pages 19-49. Springer, 2000.

5. Vasek Chvátal. Linear programming. Freeman, New York (N. Y.), 1983. Reprints : 1999, 2000, 2002

6. T. Fahle and M. Sellmann. Cost-based filtering for the constrained knapsack problem. Annals of Operations Research, (115):73-93, 2002.

7. Filippo Focacci, Andrea Lodi, and Michela Milano. Cost-based domain filtering. In International Conference on Principles and Practice of Constraint Programming, pages 189-203. Springer, 1999.

8. Filippo Focacci, Andrea Lodi, Michela Milano, and Daniele Vigo. Solving TSP through the integration of $\mathrm{OR}$ and $\mathrm{CP}$ techniques. Electronic notes in discrete mathematics, 1:13-25, 1999.

9. Filippo Focacci, Michela Milano, and Andrea Lodi. Solving TSP with time windows with constraints. In Proceedings of the 1999 international conference on Logic programming, pages 515-529. Massachusetts Institute of Technology, 1999.

10. Diego Klabjan. A new subadditive approach to integer programming. In Integer Programming and Combinatorial Optimization, pages 384-400. Springer, 2002.

11. Andrea Lodi. Mixed integer programming computation. In 50 Years of Integer Programming 1958-2008, pages 619-645. Springer, 2010.

12. Ashutosh Mahajan. Presolving mixed-integer linear programs. Wiley Encyclopedia of Operations Research and Management Science, 2010.

13. George L Nemhauser and Laurence A Wolsey. Integer programming and combinatorial optimization. Wiley, Chichester. GL Nemhauser, MWP Savelsbergh, GS Sigismondi (1992). Constraint Classification for Mixed Integer Programming Formulations. COAL Bulletin, 20:8-12, 1988.

14. P. Refalo. Linear Formulation of Constraint Programming Models and Hybrid Solver. In Principles and Practice of Constraint Programming-CP2000, Lecture Notes in Computer Science, pages 369-383. Springer-Verlag, 2000.

15. Meinolf Sellmann. Theoretical Foundations of CP-Based Lagrangian Relaxation, pages 634-647. Springer Berlin Heidelberg, Berlin, Heidelberg, 2004.

16. Erlendur S. Thorsteinsson and Greger Ottosson. Linear relaxations and reducedcost based propagation of continuous variable subscripts. Annals of Operations Research, 115(1):15-29, 2002.

17. Laurence A Wolsey. Integer programming john wiley \& sons. New York, NY, 4, 1998.

18. Tallys Yunes, Ionuţ D. Aron, and J. N. Hooker. An integrated solver for optimization problems. Operations Research, 58(2):342-356, 2010. 